\title{
Low-Diffusion Rotated Upwind Schemes, Multigrid and Defect Correction for Steady, Multi-Dimensional Euler Flows
}

by

\author{
Barry Koren
}

\begin{abstract}
Two simple, multi-dimensional upwind discretizations for the steady Euler equations are derived, with the emphasis lying on both a good accuracy and a good solvability. The multi-dimensional upwinding consists of applying a one-dimensional Riemann solver with a locally rotated left and right state, the rotation angle depending on the local flow solution. First, a scheme is derived for which smoothing analysis of point Gauss-Seidel relaxation shows that despite its rather low numerical diffusion, it still enables a good acceleration by multigrid. Next, a scheme is derived which has not any numerical diffusion in crosswind direction, and of which convergence analysis shows that its corresponding discretized equations can be solved efficiently by means of defect correction iteration with in the inner multigrid iteration the first scheme. For the steady, two-dimensional Euler equations, numerical experiments are performed for some supersonic test cases with an oblique contact discontinuity. The numerical results are in good agreement with the theoretical predictions. Comparisons are made with results obtained by a standard, grid-aligned upwind scheme. The grid-decoupled results obtained are promising.
\end{abstract}

Note: This work was supported by the European Space Agency, through Avions Marcel Dassault - Bréguet Aviation.

\section{INTRODUCTION}

In the present paper, for the steady, 2-D Euler equations in a cell-centered finite volume context, we develop a multi-D upwind method with some appropriate balance between crosswind diffusion and efficiency. The steady equations will be solved directly. Herewith, for obtaining a good efficiency, we rely on nonlinear multigrid (multigrid-Newton) iteration. As the smoothing technique for the multigrid iteration, we prefer to apply point GaussSeidel relaxation, using the exact differential matrices (exact Newton). The latter requires the cell face fluxes to be continuously differentiable. If multigrid does not easily meet our standards, we will not try to repair it, but - instead - we will rely on defect correction iteration as an additional iteration. The multi-D upwind schemes to be considered here will be very simple schemes only. They will use neither decoupling of the Euler equations (as in $[5,10]$ ), nor rotated fluxes (as in $[1,8]$ ). The schemes will be based on rotated left and right states solely. Per cell face, just as with grid-aligned upwind schemes, only a single numerical fiux is computed: that normal to the cell face. The numerical flux function to be applied should allow a good resolution of both oblique shock waves and oblique contact discontinuities, fixing choices to flux difference splitting schemes. Given the good experience with Osher's scheme [9] in combination with multigrid - (exact) Newton [4], here we will apply the latter flux difference splitting scheme. 
The contents of the paper is as follows. First, in section 2, to set a frame of reference, on the basis of a linear, scalar model equation, an accuracy analysis and a simple solvability analysis are performed for the standard, grid-aligned first-order upwind scheme. Then, two multi-D upwind schemes are derived. Next, in section 3, on the basis of the same model equation, solvability properties of the two schemes derived in section 2 are analyzed. These analyses will be Fourier analyses. Finally, in section 4, the theoretical results found in the previous sections are verified for some steady, fully supersonic Euler flows on the unit square.

\section{DERIVATION OF GRID-DECOUPLED UPWIND SCHEMES}

We will try to derive positive, grid-decoupled upwind schemes which have a low crosswind diffusion (preferably zero), and which (hopefully) still have enough artificial diffusion in characteristic direction to preserve good solvability. An important property required from these schemes is that their stencils are as compact as possible. The motivation for compactness is to avoid: non-consistent boundary condition treatments and - if possible - nonpositivity. Compactness will result in a close relationship with the grid-aligned first-order upwind scheme, which therefore will be used as the main reference for comparison. Another property strived for is continuous differentiability, this because of the intended application of exact Newton iteration. The schemes will be investigated on the basis of the linear, scalar, 2-D model equation

$$
\cos \phi \frac{\partial u}{\partial x}+\sin \phi \frac{\partial u}{\partial y}=0, \quad 0 \leqslant \phi \leqslant \pi / 2
$$

with $\phi$ the angle made by the characteristic direction and the $x$-axis. Discretization of (2.1) on a square, cell-centered finite volume grid yields

$$
\cos \phi\left(u_{i+1 / 2, j}-u_{i-1 / 2, j}\right)+\sin \phi\left(u_{i, j}+1 / 2-u_{i, j-1 / 2}\right)=0,
$$

where the non-integer indices refer to the cell faces in between the (integer-indexed) cell centers.

In the grid-aligned first-order upwind scheme, given the being positive of $\cos \phi$ and $\sin \phi$, for the cell face states we take

$$
\left[\begin{array}{l}
u_{i+1 / 2, j} \\
u_{i, j}+1 / 2
\end{array}\right]=\left[\begin{array}{l}
u_{i, j} \\
u_{i, j}
\end{array}\right], \quad 0 \leqslant \phi \leqslant \pi / 2 .
$$

Similar choices are made for $u_{i-1 / 2, j}$ and $u_{i, j-1 / 2}$. Substituting these cell face states into (2.2) and applying next truncated Taylor series expansions, with $h$ as the mesh size, the following modified equation may be derived:

$$
\cos \phi \frac{\partial u}{\partial x}+\sin \phi \frac{\partial u}{\partial y}-\frac{h}{2}\left[\cos \phi \frac{\partial^{2} u}{\partial x^{2}}+\sin \phi \frac{\partial^{2} u}{\partial y^{2}}\right]=\mathrm{O}\left(h^{2}\right), \quad 0 \leqslant \phi \leqslant \pi / 2 .
$$

By transformation to characteristic coordinates, (2.4) becomes

$$
\begin{gathered}
\frac{\partial u}{\partial s}-\frac{h}{2}\left[\left(\cos ^{3} \phi+\sin ^{3} \phi\right) \frac{\partial^{2} u}{\partial s^{2}}-2 \cos \phi \sin \phi(\cos \phi-\sin \phi) \frac{\partial^{2} u}{\partial s \partial n}+\right. \\
\left.\cos \phi \sin \phi(\cos \phi+\sin \phi) \frac{\partial^{2} u}{\partial n^{2}}\right]=\mathrm{O}\left(h^{2}\right), \quad 0 \leqslant \phi \leqslant \pi / 2 .
\end{gathered}
$$

From (2.5) now, it appears that for the grid-aligned first-order upwind scheme, zerocrosswind diffusion (i.e. both $\cos \phi \sin \phi(\cos \phi-\sin \phi)=0$ and $\cos \phi \sin \phi(\cos \phi+\sin \phi)=0)$ occurs only in case of $\phi=0$ or $\phi=\pi / 2$, i.e. in case of grid-alignment of the characteristic direction. 
For the sake of comparisons to be made hereafter, in Fig. 2.1a we give the distributions of the diffusion coefficients from (2.5), over the complete range of $\phi$ considered. In here and also in the following, $\mu_{s s}, \mu_{s n}$ and $\mu_{n n}$ denote the coefficients of $\partial^{2} u / \partial s^{2}, \partial^{2} u / \partial s \partial n$ and $\partial^{2} u / \partial n^{2}$, respectively.

As opposed to the poor accuracy properties, the smoothing properties of point GaussSeidel relaxation applied to the grid-aligned first-order upwind system are known to be good. The fact that these smoothing properties are at least not bad is reflected by the fact that the discretization is positive, which clearly appears from the stencil

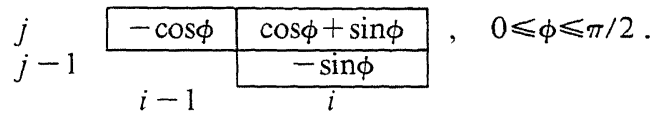

2.1. A positive, continuously differentiable scheme

The most compact, grid-decoupled upwind schemes use

$$
\left[\begin{array}{l}
u_{i+1 / 2, j} \\
u_{i, j}+1 / 2
\end{array}\right]=\left[\begin{array}{l}
\delta_{1}(\phi) u_{i, j}+\left[1-\delta_{1}(\phi)\right] u_{i, j-1} \\
\delta_{2}(\phi) u_{i, j}+\left[1-\delta_{2}(\phi)\right] u_{i-1, j}
\end{array}\right], \quad 0 \leqslant \delta_{1}(\phi), \delta_{2}(\phi) \leqslant 1, \quad 0 \leqslant \phi \leqslant \pi / 2 .
$$

The coefficients $\delta_{1}(\phi)$ and $\delta_{2}(\phi)$, whose choice determines the scheme, are taken in the range $[0,1]$ in order to prevent unphysical features such as e.g. a negative density. The stencil corresponding with $(2.7)$ reads

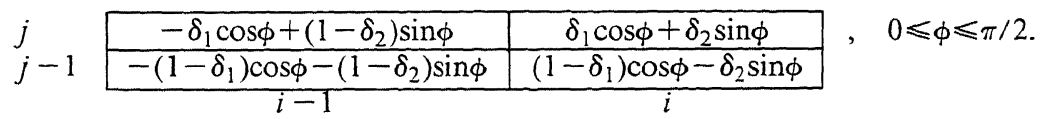

A natural requirement imposed to the discretization is that of symmetry with respect to $\phi=\pi / 4 ;$ in formula:

$$
\delta_{2}(\phi)=\delta_{1}(\pi / 2-\phi), \quad 0 \leqslant \phi \leqslant \pi / 2 .
$$

The modified equation corresponding with (2.7) reads in characteristic coordinates

$$
\begin{aligned}
\frac{\partial u}{\partial s}-\frac{h}{2}[ & \left.(1+\cos \phi \sin \phi)(\cos \phi+\sin \phi)-2\left(\delta_{1} \cos \phi+\delta_{2} \sin \phi\right) \cos \phi \sin \phi\right) \frac{\partial^{2} u}{\partial s^{2}}+ \\
& 2(\cos \phi-\sin \phi)\left(1+\cos \phi \sin \phi-\left(\delta_{1} \cos \phi+\delta_{2} \sin \phi\right)(\cos \phi+\sin \phi)\right] \frac{\partial^{2} u}{\partial s \partial n}+ \\
& \left.2 \cos \phi \sin \phi\left(\left(\delta_{1}-1 / 2\right) \cos \phi+\left(\delta_{2}-1 / 2\right) \sin \phi\right) \frac{\partial^{2} u}{\partial n^{2}}\right]=O\left(h^{2}\right), \quad 0 \leqslant \phi \leqslant \pi / 2
\end{aligned}
$$

Following (2.10), $\mu_{s n}=0$ and $\mu_{n n}=0$ lead to respectively

$$
\begin{array}{ll}
\delta_{1} \cos \phi+\delta_{2} \sin \phi=\frac{1+\cos \phi \sin \phi}{\cos \phi+\sin \phi}, & 0 \leqslant \phi \leqslant \pi / 2, \\
\delta_{1} \cos \phi+\delta_{2} \sin \phi=1 / 2(\cos \phi+\sin \phi), & 0 \leqslant \phi \leqslant \pi / 2,
\end{array}
$$

which clearly is an inconsistent system of equations, leading to the following theorem:

THEOREM (2.1)

No most compact grid-decoupled upwind scheme exists for which both $\mu_{s n}=0$ and $\mu_{n n}=0$. 
Next, concerning crosswind diffusion, we only require $\mu_{n n}$ to be zero, i.e. we assume (2.11b) to hold solely. Further, following (2.8), positivity can be expressed as:

$$
\left(\delta_{1} \cos \phi+\delta_{2} \sin \phi\right)\left(\begin{array}{c}
1 \\
1 \\
1 \\
-1
\end{array}\right)>\left(\begin{array}{c}
0 \\
\sin \phi \\
\cos \phi \\
-\cos \phi-\sin \phi
\end{array}\right), \quad 0 \leqslant \phi \leqslant \pi / 2
$$

It appears that the system $(2.11 \mathrm{~b})-(2.12)$ is inconsistent as well, leading to the theorem:

THEOREM (2.2)

No most compact grid-decoupled upwind scheme exists which is positive and for which $\mu_{n n}=0$.

Finally, we require $\mu_{s n}=0$ to hold (i.e. (2.11a)) in combination with (2.12). It can be verified that this system is consistent. Assuming the form $\delta_{1}(\phi)=(\cos \phi+a \sin \phi) /(\cos \phi+\sin \phi), a$ being a constant, with symmetry requirement (2.9) we get $\delta_{2}(\phi)=(\sin \phi+a \cos \phi) /(\sin \phi+\cos \phi)$. Substitution of these forms of $\delta_{1}(\phi)$ and $\delta_{2}(\phi)$ into (2.11a) yields $a=1 / 2$, so

$$
\left(\begin{array}{l}
u_{i+1 / 2, j} \\
u_{i, j}+1 / 2
\end{array}\right]=\frac{1}{\cos \phi+\sin \phi}\left[\begin{array}{l}
(\cos \phi+1 / 2 \sin \phi) u_{i, j}+1 / 2 \sin \phi u_{i, j-1} \\
(\sin \phi+1 / 2 \cos \phi) u_{i, j}+1 / 2 \cos \phi u_{i-1, j}
\end{array}\right), \quad 0 \leqslant \phi \leqslant \pi / 2,
$$

which gives the (positive) stencil

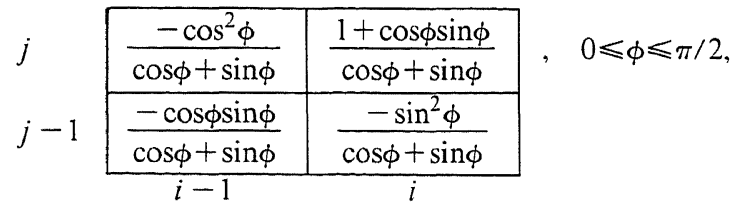

and the modified equation

$$
\frac{\partial u}{\partial s}-\frac{h}{2}\left[\frac{1+\cos \phi \sin \phi}{\cos \phi+\sin \phi} \frac{\partial^{2} u}{\partial s^{2}}+\frac{\cos \phi \sin \phi}{\cos \phi+\sin \phi} \frac{\partial^{2} u}{\partial n^{2}}\right]=\mathrm{O}\left(h^{2}\right), \quad 0 \leqslant \phi \leqslant \pi / 2 .
$$

In Fig. 2.1b we give the graph of the present scheme's diffusion properties. The scheme's crosswind diffusion is significantly lower than that of the grid-aligned first-order upwind scheme (Fig. 2.1a). The lower crosswind diffusion in combination with the properties of positivity and continuous differentiability makes that scheme (2.13) is possibly more appropriate for our present multigrid purposes than the grid-aligned first-order upwind scheme (2.3).

\subsection{A zero-crosswind diffusion scheme}

Because of the limitation of the most compact, grid-decoupled upwind schemes with respect to the elimination of all crosswind diffusion (the limitation expressed by THEOREM (2.1)), in this section, we will consider wider stencils. To start with, we consider a situation with small $\phi$. Still striving for compactness, the extrapolation is done from the nearest lines connecting two neighboring cell center states, also avoiding negative coefficients herewith:

$$
\left(\begin{array}{l}
u_{i+1 / 2, j} \\
u_{i, j}+1 / 2
\end{array}\right]=\left[\begin{array}{c}
\delta_{1}(\phi) u_{i, j}+\left[1-\delta_{1}(\phi)\right] u_{i, j-1} \\
\delta_{2}(\phi) u_{i-1, j}+\left[1-\delta_{2}(\phi)\right] u_{i-1, j+1}
\end{array}\right], \quad 0 \leqslant \delta_{1}(\phi), \delta_{2}(\phi) \leqslant 1, \quad 0 \leqslant \phi \leqslant \phi_{u p},
$$



with the (small) upper bound $\phi_{u p}$ not yet fixed. With (2.16), we find the following modified
equation

$$
\begin{aligned}
\frac{\partial u}{\partial s}-\frac{h}{2}[ & \left(\cos ^{3} \phi+2\left(1-\delta_{1}\right) \cos ^{2} \phi \sin \phi+2 \cos \phi \sin ^{2} \phi+\left(2 \delta_{2}-1\right) \sin ^{3} \phi\right) \frac{\partial^{2} u}{\partial s^{2}}+ \\
& 2\left(\left(1-\delta_{1}\right) \cos ^{3} \phi+\left(\delta_{1}+2 \delta_{2}-2\right) \cos \phi \sin ^{2} \phi-\sin ^{3} \phi\right) \frac{\partial^{2} u}{\partial s \partial n}+ \\
& \left.\cos \phi \sin \phi\left[\left(2 \delta_{1}+2 \delta_{2}-3\right) \cos \phi-\sin \phi\right) \frac{\partial^{2} u}{\partial n^{2}}\right]=\mathrm{O}\left(h^{2}\right), \quad 0 \leqslant \phi \leqslant \phi_{u p} .
\end{aligned}
$$

From this, it follows that no crosswind diffusion occurs for $\delta_{1}(\phi)=1$, $\delta_{2}(\phi)=1 / 2(1+\tan \phi), 0 \leqslant \phi \leqslant \pi / 4$, where the indicated $\phi$-range (with $\phi_{u p}=\pi / 4$ ) is that for which negative coefficients are just avoided. Taking for the remaining subrange the symmetrical counterpart of (2.16):

$$
\left(\begin{array}{c}
u_{i+1 / 2, j} \\
u_{i, j}+1 / 2
\end{array}\right)=\left(\begin{array}{c}
\delta_{1}(\phi) u_{i, j-1}+\left[1-\delta_{1}(\phi)\right] u_{i+1, j-1} \\
\delta_{2}(\phi) u_{i, j}+\left[1-\delta_{2}(\phi)\right] u_{i-1, j}
\end{array}\right), \quad \pi / 4 \leqslant \phi \leqslant \pi / 2
$$

with symmetry condition (2.9) we get $\delta_{1}(\phi)=1 / 2(1+\operatorname{cotan} \phi), \delta_{2}(\phi)=1, \pi / 4 \leqslant \phi \leqslant \pi / 2$. Summarizing, we have derived as expressions for the cell face states:

$$
\begin{gathered}
{\left[\begin{array}{l}
u_{i+1 / 2, j} \\
u_{i, j}+1 / 2
\end{array}\right]=\left(\begin{array}{c}
u_{i, j} \\
1 / 2(1+\tan \phi) u_{i-1, j}+1 / 2(1-\tan \phi) u_{i-1, j+1}
\end{array}\right), \quad 0 \leqslant \phi \leqslant \pi / 4,} \\
{\left[\begin{array}{l}
u_{i+1 / 2, j} \\
u_{i, j}+1 / 2
\end{array}\right]=\left(\begin{array}{c}
1 / 2(1+\operatorname{cotan} \phi) u_{i, j-1}+1 / 2(1-\operatorname{cotan} \phi) u_{i+1, j-1} \\
u_{i, j}
\end{array}\right], \quad \pi / 4 \leqslant \phi \leqslant \pi / 2,}
\end{gathered}
$$

as corresponding stencils:

$$
\begin{aligned}
& \begin{array}{l|c|c|}
j+1 & 1 / 2 \sin \phi(1-\tan \phi) & \\
\cline { 2 - 3 } j-1 & \sin \phi(\tan \phi-\operatorname{cotan} \phi) & \cos \phi \\
\cline { 2 - 3 } & \multicolumn{1}{c}{, \quad 0 \leqslant \phi \leqslant \pi / 4 \sin \phi(1+\tan \phi)} &
\end{array}
\end{aligned}
$$

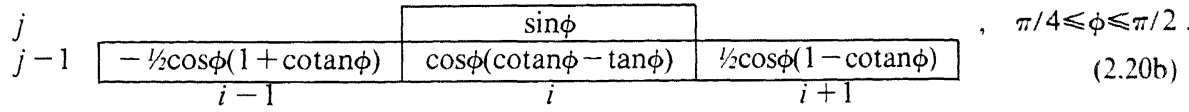

and as modified equations:

$$
\begin{aligned}
& \frac{\partial u}{\partial s}-\frac{h}{2} \frac{1}{\cos \phi} \frac{\partial^{2} u}{\partial s^{2}}=\mathrm{O}\left(h^{2}\right), \quad 0 \leqslant \phi \leqslant \pi / 4, \\
& \frac{\partial u}{\partial s}-\frac{h}{2} \frac{1}{\sin \phi} \frac{\partial^{2} u}{\partial s^{2}}=\mathrm{O}\left(h^{2}\right), \quad \pi / 4 \leqslant \phi \leqslant \pi / 2 .
\end{aligned}
$$

A geometric interpretation of scheme (2.19) is given in [3]. In Fig. 2.1c, we give the graph with its diffusion coefficients. Unfortunately, from $(2.20)$ it appears that the scheme is nonpositive. A favorable property of the scheme is its simplicity. 


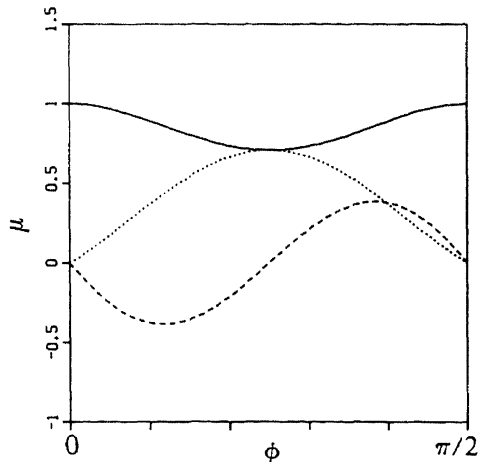

a. Grid-aligned first-order upwind scheme (2.3).

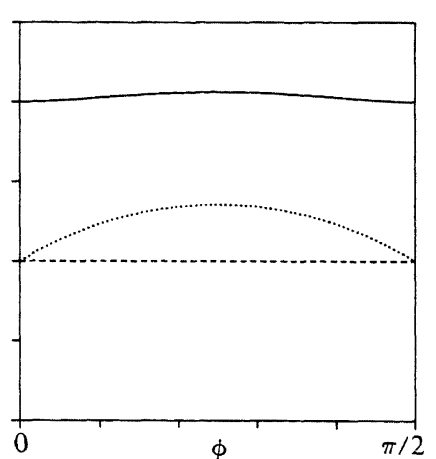

b. Continuously differentiable scheme (2.13).

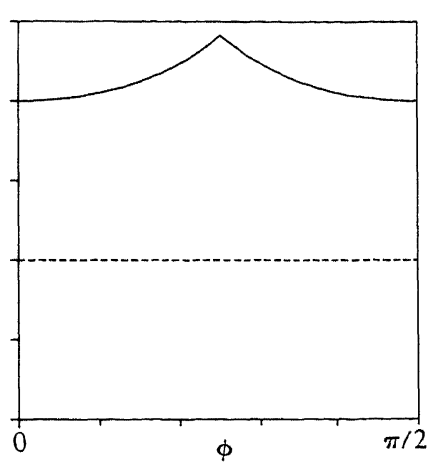

c. Zero-crosswind diffusion scheme (2.19).

Fig. 2.1. Diffusion coefficients modified equation

$$
\left(\mu_{s s}:-, \mu_{s n}:-\cdots---, \mu_{n n}: \ldots \ldots . .\right) \text {. }
$$

\section{ANALYSIS OF MULTIGRID ITERATION AND DEFECT CORRECTION ITERATION}

In the present section, we will analyze some possible solution methods for the two griddecoupled schemes derived in the previous section. The analyses will be done again for model equation (2.1) on a square, cell-centered finite volume grid. First, the smoothing behavior of point Gauss-Seidel relaxation will be investigated, both for scheme (2.13) and scheme (2.19). (Though we do not expect the latter scheme to be sufficiently dissipative to allow a successful application of multigrid iteration, for sake of certainty we do consider its smoothing behavior.) The smoothing properties found will be compared with those of the grid-aligned first-order upwind scheme (2.3). If analysis shows that multigrid iteration applied directly to the zero-crosswind diffusion operator does not work, to solve the corresponding system of discretized equations, we will rely on defect correction iteration. We already have good experience with defect correction iteration, in efficiently solving higher-order grid-aligned Euler and Navier-Stokes equations (see [2,6] and [7], respectively).

\subsection{Smoothing analysis of point Gauss-Seidel relaxation}

Four different relaxation sweep directions are considered: downwind, upwind and twice crosswind, each of those four with the $i$-loop as the inner sweep-loop, and each for the complete range of $\phi$ considered: [0, $\pi / 2]$. To apply smoothing analysis, denoting the number of sweeps performed by $n$, we introduce: (i) the iteration error

$$
\delta_{i, j}^{n} \equiv u_{i, j}^{*}-u_{i, j}^{n} \text {, }
$$

with $u_{i, j}^{*}$ the converged numerical solution in finite volume $i, j$, and (ii) the Fourier form

$$
\delta_{i, j}^{n}=D \rho^{n} e^{\mathrm{i}\left(\theta_{1}+\theta_{2}\right)},
$$

with $D$ constant, $\rho$ the amplification factor, and $\theta_{1} \equiv \omega_{1} h$ and $\theta_{2} \equiv \omega_{2} h, \omega_{1}$ and $\omega_{2}$ being the error mode in $i$ - and $j$-direction, respectively. In Fig. 3.1, results of the smoothing analysis are given for successively: grid-aligned first-order upwind scheme (2.3), continuously differentiable scheme (2.13) and zero-crosswind diffusion scheme (2.19). In here, the smoothing factor $\rho_{s}$ is defined as

$$
\rho_{s} \equiv \sup \left|\rho\left(\theta_{1}, \theta_{2}\right)\right|, \quad\left(\left|\theta_{1}\right|,\left|\theta_{2}\right|\right) \in\left\{[0, \pi] \times[0, \pi]|| \theta_{1}|\in[\pi / 2, \pi] \vee| \theta_{2} \mid \in[\pi / 2, \pi]\right\} .
$$




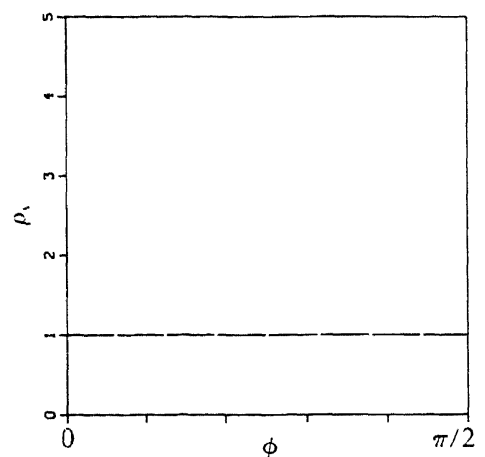

a. Grid-aligned first-order upwind scheme (2.3).

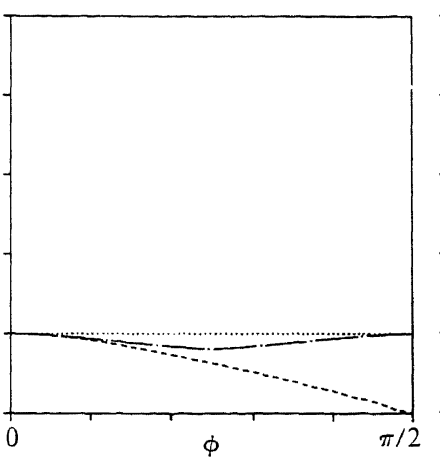

b. Continuously differentiable scheme (2.13).

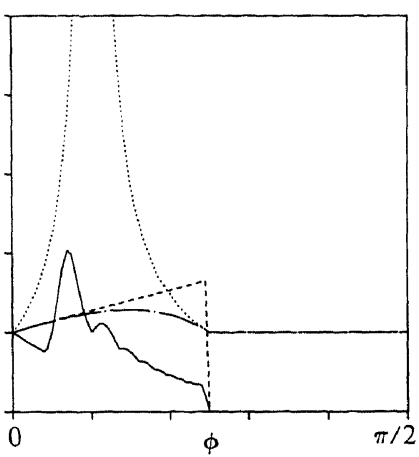

c. Zero-crosswind diffusion scheme (2.19).

Fig. 3.1. Smoothing factors point Gauss-Seidel relaxation, with $i$-loop as inner loop

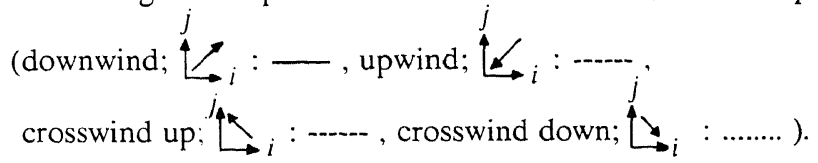

It appears that the zero-crosswind diffusion scheme does not enable a successful application of multigrid with point Gauss-Seidel relaxation as the smoother (Fig. 3.1c). Notice that even for the corresponding downwind relaxation sweep, smoothing is not guaranteed over the complete range of $\phi$ considered. As opposed to this, for the positive, continuously differentiable scheme we do have smoothing (Fig. 3.1b).

To find a suitable solution method for zero-crosswind diffusion scheme (2.19), in the following, we will study the convergence properties of defect correction iteration with positive, continuously differentiable scheme (2.13) as the approximate scheme in the inner multigrid iteration. For comparison, a similar study will also be made with grid-aligned first-order upwind scheme (2.3) as the approximate scheme.

\subsection{Convergence analysis of defect correction iteration}

Denoting the zero-crosswind diffusion operator by $L_{h}^{+}$, defect correction iteration reads

$$
L_{h}\left(u_{h}^{n+1}\right)=L_{h}\left(u_{h}^{n}\right)-L_{h}^{+}\left(u_{h}^{n}\right), \quad n=0,1, \ldots, N,
$$

with $L_{h}$ denoting the positive operator to be inverted, and with the index $n$ denoting the iteration counter. From (3.4), it is clear that the closer the resemblance between the target operator $L_{h}^{+}$and the approximate operator $L_{h}$, the better the convergence of the defect correction iteration. Hence, in this respect, the best convergence of (3.4) is expected from the approach with (2.13) as the approximate scheme. Introducing, as before, the iteration error (3.1) in its Fourier form (3.2), we can write for the amplification factor

$$
\rho\left(\theta_{1}, \theta_{2}\right)=1-L_{h}^{-1}\left(\theta_{1}, \theta_{2}\right) L_{h}^{+}\left(\theta_{1}, \theta_{2}\right) .
$$

In Fig. 3.2, for each of the two approximate schemes (2.3) and (2.13), and for successively $\phi=0.1 \pi, 0.2 \pi, 0.3 \pi$ and $0.4 \pi$, we give the distributions of the convergence factor $\rho_{c}$;

$$
\rho_{c} \equiv\left|\rho\left(\theta_{1}, \theta_{2}\right)\right|, \quad\left(\left|\theta_{1}\right|,\left|\theta_{2}\right|\right) \in\{[0, \pi] \times[0, \pi]\} .
$$



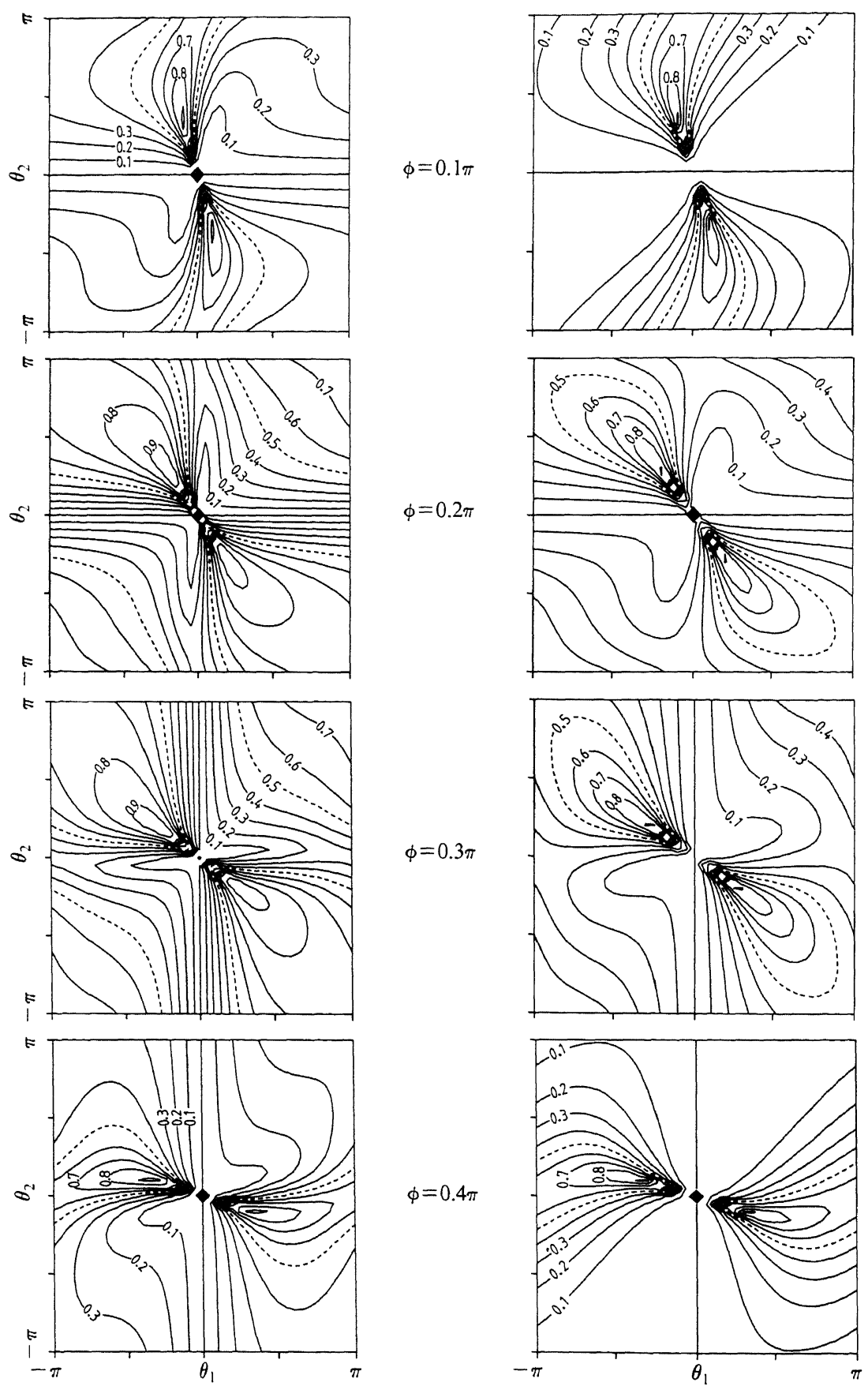

a. Grid-aligned first-order upwind scheme (2.3)

b. Continuously differentiable scheme (2.13) as approximate scheme. as approximate scheme.

Fig. 3.2. Convergence factor distributions defect correction iteration. 
In all graphs of Fig. 3.2, the dashed iso-lines correspond with $\rho_{c}=0.5$. It appears that, for all four values of $\phi$ considered, scheme (2.13) as the approximate scheme gives better convergence factor distributions indeed.

\section{NUMERICAL RESUlts}

To investigate some theoretical results found in the previous sections, in this section, for a perfect gas with $\gamma=1.4$, we perform numerical experiments for a set of fully supersonic Euler flows with oblique contact discontinuity (Fig. 4.1a). The discontinuity is considered for the flow angles $\phi=0.1 \pi, 0.2 \pi, 0.3 \pi$ and $0.4 \pi$. The flows are computed on the $32 \times 32$-grid given in Fig. 4.1b. In all cases - for simplicity - at each of the four boundaries, the exact solution is imposed (overspecification). The multigrid method applied for all cases is nonlinear multigrid with $\mathrm{V}$-cycles, and with per level a single pre- and post-relaxation sweep only. In all cases, the coarsest grid considered is a $2 \times 2$-grid. Further, in all cases we take as the initial solution: the solution with $q=q^{L}$ (the $q^{L}$ from Fig. 4.1a) uniformly constant over the complete domain. For both grid-decoupled upwind schemes derived before, as the angle to be considered at each cell face, we take the streamline angle. In [3], we give a physically proper way of computing this angle.

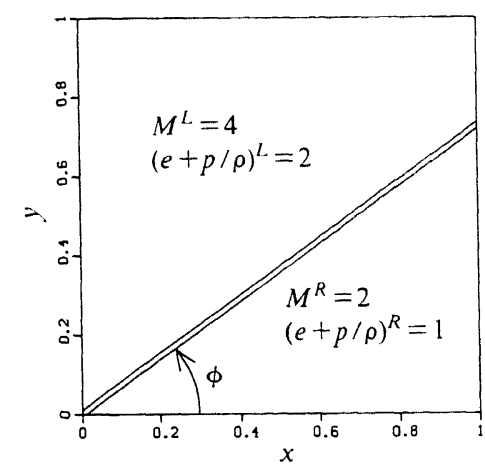

a. Flow with contact discontinuity $(\phi=0.1 \pi, 0.2 \pi, 0.3 \pi, 0.4 \pi, p=1)$.

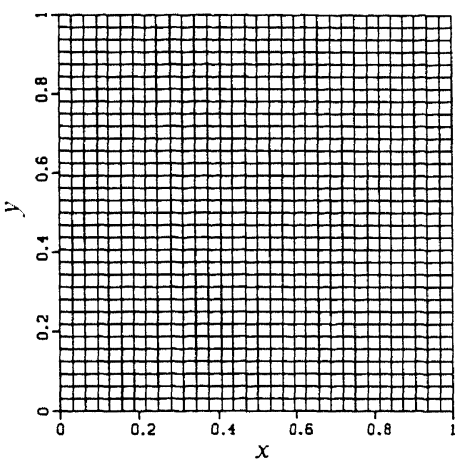

b. Finest grid $(32 \times 32)$.

Fig. 4.1. Test case on unit square.

4.1. Results positive, continuously differentiable scheme

In Fig. 4.2 we first give, on top of each other, the reference results obtained for $\phi=0.1 \pi, 0.2 \pi, 0.3 \pi$ and $0.4 \pi$ with the grid-aligned first-order upwind scheme; in Fig. $4.2 \mathrm{a}$ the multigrid convergence histories and in Fig. $4.2 \mathrm{~b}$ the enthalpy $(e+p / \rho)$ distributions. The iso-enthalpy values considered in these and all following enthalpy distributions are: $1.1,1.2,1.3, \cdots, 1.9$. Because of the severe smearing of the grid-aligned first-order upwind scheme, hardly any distinction can be made between the four solutions. Notice that the layers along $x=1$ and $y=1$, in Fig. 4.2b and all following enthalpy graphs, are only due to the overspecification. 


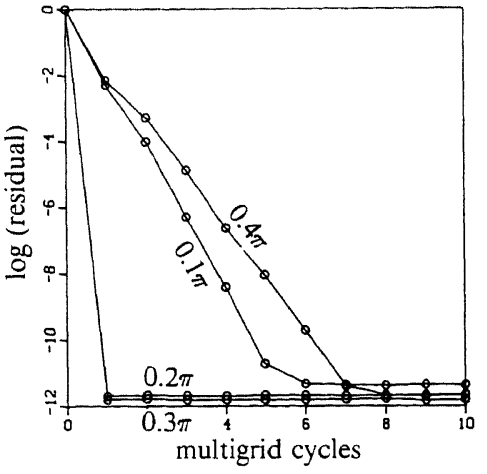

a. Multigrid convergence histories.

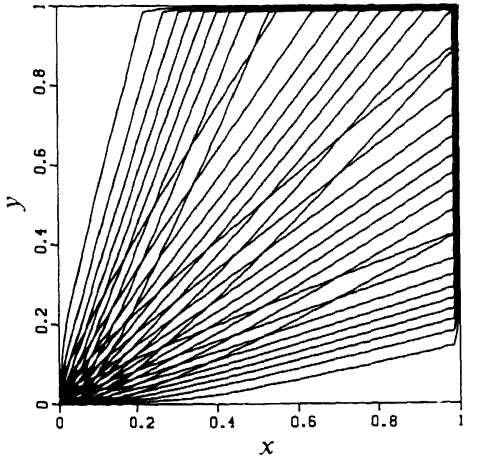

b. Enthalpy distributions.

Fig. 4.2. Results grid-aligned first-order upwind scheme.

In Fig. 4.3 we give the similar results obtained with the grid-decoupled, positive, continuously differentiable scheme. Though not as very fast as the reference convergence in Fig. 4.2a, fortunately, the present scheme's multigrid convergence is still fast. Though clearly more accurate than the reference distributions in Fig. 4.2b, the present enthalpy distributions are still insufficiently accurate.

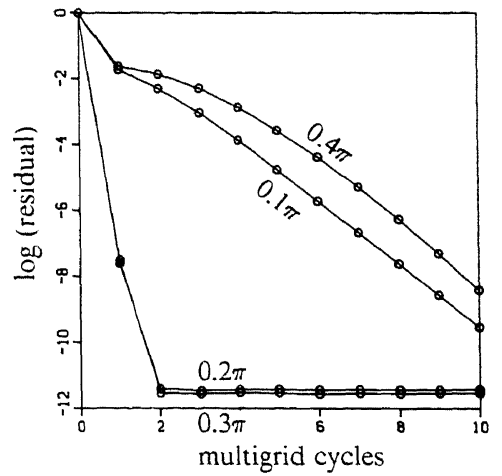

a. Multigrid convergence histories.

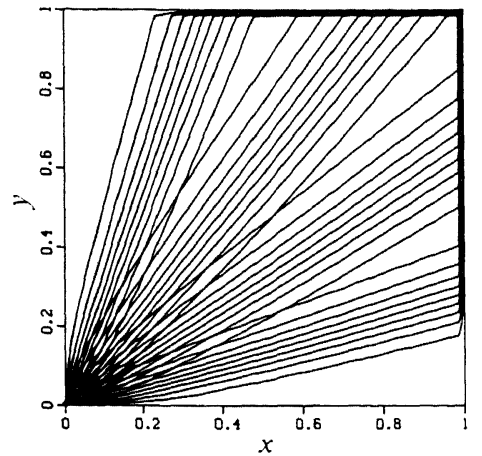

b. Enthalpy distributions.

Fig. 4.3. Results positive, continuously differentiable scheme. 
4.2. Results zero-crosswind diffusion scheme

In Fig. 4.4 we give the enthalpy distributions for the zero-crosswind diffusion scheme, as obtained after successively 1,2 and 10 defect correction cycles (with per defect correction cycle a single nonlinear multigrid cycle only). The distributions in Fig. 4.4c appear to be almost free of crosswind diffusion. (In [3] we also give the enthalpy distributions obtained with a third-order accurate, grid-aligned upwind scheme. All four enthalpy distributions in Fig. 4.4c appear to be even less diffusive than those of that third-order accurate scheme.) Though the being non-positive of the zero-crosswind diffusion scheme allows solutions with spurious oscillations, its distributions are still monotone. Concerning the convergence rate of the defect correction iteration, we are of the opinion that this is good.

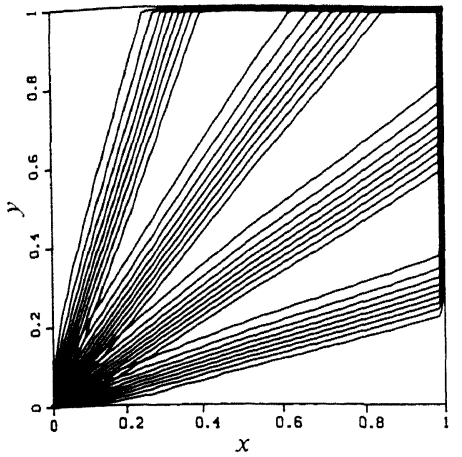

a. After 1 st cycle.

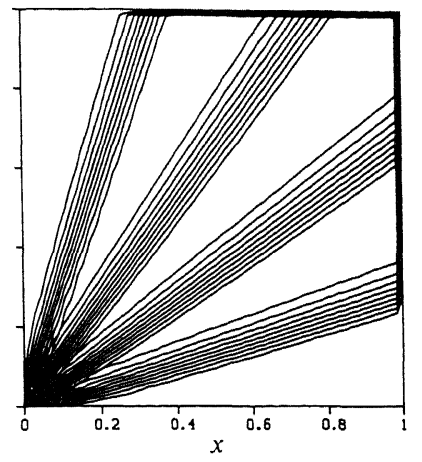

b. After 2 nd cycle.

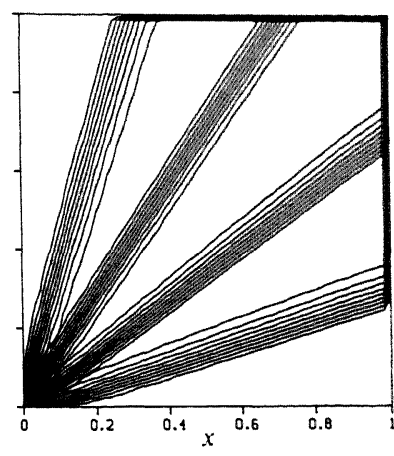

c. After 10 th cycle.

Fig. 4.4. Convergence history defect correction iteration, enthalpy distributions zero-crosswind diffusion scheme.

\begin{abstract}
5. CONCLUSIONS
In the present paper, for multi-D Euler equations, two promising multi-D upwind schemes have been derived; a positive, continuously differentiable scheme and a zero-crosswind diffusion scheme. Both schemes are based on a one-dimensional Riemann solver. Their multi-D nature is simply realized through a local, solution-dependent rotation of the left and right Riemann state, allowing to keep the number of numerical flux computations per cell face at one only. Good efficiency is strived for by means of nonlinear multigrid iteration and (if necessary) defect correction iteration. The accuracy and efficiency of the numerical results obtained are promising. One important result obtained is that when applying the positive, continuously differentiable scheme, for flows with contact discontinuities, the performance of nonlinear multigrid with point Gauss-Seidel relaxation as the smoother, is very good. Another important result is that, again for flows with contact discontinuities, the solutions of the zero-crosswind diffusion scheme appear to be practically free of crosswind diffusion indeed. Moreover, their computation by means of defect correction iteration, with the grid-decoupled, positive, continuously differentiable scheme as the approximate scheme, is efficient.
\end{abstract}


REFERENCES

1. DAvis, S.F., A rotationally biased upwind difference scheme for the Euler equations, $J$. Comput. Phys. 56 (1984), 65-92.

2. Hemker, P.W., Defect correction and higher order schemes for the multi grid solution of the steady Euler equations, Proceedings 2nd European Conference on Multigrid Methods, Cologne, 1985, Lecture Notes in Mathematics, No. 1228, Springer, Berlin, 1986, 149-165.

3. Hemker, P.W., Koren, B. ANd VAN LeER, B., Low-diffusion upwind schemes, multigrid and defect correction for steady, multi-dimensional Euler flows, Center for Mathematics and Computer Science, Amsterdam, NM-Report (in preparation).

4. HeMker, P.W. AND SPEKREIJSE, S.P., Multiple grid and Osher's scheme for the efficient solution of the steady Euler equations, Appl. Numer. Math. 2 (1986), 475-493.

5. Hirsch, Ch., LaCor, C. AND DeCONINCK, H., Convection algorithms based on a diagonalization procedure for the multidimensional Euler equations, AIAA Paper 87-1163 (1987).

6. KOREN, B., Defect correction and multigrid for an efficient and accurate computation of airfoil flows, J. Comput. Phys. 77 (1988), 183-206.

7. Koren, B., Multigrid and defect correction for the steady Navier-Stokes equations, $J$. Comput. Phys. 87 (1990), 25-46.

8. LeVY, D.W., Powell, K.G. AND VAN Leer, B., An implementation of a gridindependent upwind scheme for the Euler equations, A IAA Paper 89-1931 (1989).

9. OSHER, S. AND SOLOMON, F., Upwind difference schemes for hyperbolic systems of conservation laws, Math. Comput. 38 (1982), 339-374.

10. RoE, P.L., Discrete models for the numerical analysis of time-dependent multidimensional gas dynamics, J. Comput. Phys. 63 (1986), 458-476.

Barry Koren

Center for Mathematics and Computer Science

P.O. Box 4079

1009 AB Amsterdam, The Netherlands 